BNL $-77034-2006-C P$

\title{
Te inclusions and their relationship to the performance of CaZnTe detectors
}

\author{
G.:A. Carini ${ }^{a^{*}}$, A. E. Bolotnikov ${ }^{\mathrm{a}}$, G. S. Camarda ${ }^{\mathrm{a}}$, Y. Cui ${ }^{\mathrm{a}}$, H. Jackson ${ }^{\mathrm{b}}$, A. \\ Burger $^{\text {b }}$, K. T. Kohman ${ }^{c}$, L. Li ${ }^{\text {d }}$, and R. B. James ${ }^{a}$. \\ ${ }^{a}$ Brookhaven National Laboratory, Upton, NY 11973 \\ ${ }^{b}$ Fisk University, Nashville, TN 37208 \\ 'Kansas State University, Manhattan, KS 66506 \\ ${ }^{\mathrm{d}}$ Yinnel Tech, Inc., South Bend; IN 46619
}

SPIE, Hard X-ray and Gamma-Ray Detectors Physics VIII

San Diego, California

August $14^{\text {th }}, 2006$

\author{
Nonproliferation and National Security Department \\ Detector Development and Testing Division \\ Brookhaven National Laboratory \\ P.O. Box 5000 \\ Upton, NY 11973-5000 \\ www.bnl.gov
}

\begin{abstract}
Notice: This manuscript has been authored by employees of Brookhaven Science Associates, LLC.under Contract No. DE-AC02-98CH10886 with the U.S. Department of Energy. The publisher by accepting the manuscript for publication acknowledges that the United States Government retains a non-exclusive, paid-up, irrevocable, world-wide license to publish or reproduce the published form of this manuscript, or allow others to do so, for United States Government purposes.
\end{abstract}

This preprint is intended for.publication in a journal or proceedings. Since changes may be made before publication; it may not be cited or reproduced without the author's permission. 


\section{DISCLAIMER}

This report was prepared as an account of work sponsored by an agency of the United: States Government. Neither the United States Government nor any agency, thereof, nor any of their employees, nor any of their contractors, subcontractors; or their employees, makes any warranty, express or implied, or assumes any legal liability or responsibility for the accuracy, completeness, or any third party's use or the results of such use of any information, apparatus, product, or process disclosed; or represents that its use would not infringe privately owned rights. Reference herein to any. specific commercial product, process, or service by trade name, trademark, manufacturer,.. or otherwise, does not necessarily constitute or imply its endorsement, recommendation, or favoring by the United States Government or any agency thereof or its contractors or subcontractors. The views and opinions of authors expressed herein do not necessarily state or reflect those of the United States Government or any agency thereof. 


\title{
Te inclusions and their relationship to the performance of $\mathrm{CdZnTe}$ detectors
}

\author{
Kohman $^{\text {c }}$ L. Li ${ }^{\text {, }}$, and R. B. James ${ }^{\mathrm{a}}$. \\ ${ }^{\text {a} B r o o k h a v e n ~ N a t i o n a l ~ L a b o r a t o r y, ~ U p t o n, ~ N Y . ~} 11973$ \\ ${ }^{\mathrm{b}}$ Fisk University, Nashville, TN 37208 \\ ${ }^{\mathrm{C}}$ Kansas State University, Manhattan, KS 66506 \\ ${ }^{d}$ Yinnel Tech, Inc., South Bend, IN 46619
}

G. A. Carini ${ }^{{ }^{*}}$, A. E. Bolotnikov ${ }^{\mathrm{a}}$, G. S. Camarda ${ }^{\mathrm{a}}$, Y. Cui ${ }^{\mathrm{a}}$, H. Jackson ${ }^{\mathrm{b}}$, A. Burger ${ }^{\mathrm{b}}$, K. T.

\begin{abstract}
Te-rich secondary phases existing in . CdZnTe (CZT) single crystals degrade the spectroscopic performance of these detectors. An unpredictable number of charges are trapped, corresponding to the abundance of these microscopic defects, thereby leading to fluctuations in the total collected charge and strongly affecting the uniformity: of charge-collection efficiency. These effects, observed in thin planar detectors, also were found to be the dominant cause of the low performance of thick detectors, wherein the fluctuations accumulate along the charge's drift path. Reducing the size of. Te inclusions from a virtual diameter of $10-20 \mu \mathrm{m}$ down to less than $5 . \mu \mathrm{m}$ already allowed us to produce Frisch-ring detectors with a resolution as good as $\sim 0.8 \%$.FWHM at $662 \mathrm{keV}$ : Understanding and modeling the mechanisms involving Te-rich secondary phases and charge loss requires systematic studies on a spatial scale never before realized: Here, we describe a dedicated beam-line recently established at BNL's National Synchrotron Light Source for characterizing semiconductor detectors along with a IR system with counting capability that permits us to correlate the concentration of defects with the devices' performances.
\end{abstract}

Keywords: $\mathrm{CdZnTe}$, Te inclusions, synchrotron radiation, gamma ray detectors

\section{INTRODUCTION}

For decades cadmium zinc telluride (CZT) has been considered as one of the most promising materials for gamma-ray detectors operable at room temperature. However, the full exploitation of this technology for producing large-volume gamma-ray spectrometers and imaging arrays has been hampered by the low yield of CZT crystals, and their consequent high cost. In fact, because of deficiencies in the quality of the material, commercial high-resolution spectrometers still are limited to ones of relatively small dimensions $\left(<2-3 \mathrm{~cm}^{3}\right)$. Achieving the goal of inexpensive but efficient

"carini@bnl.gov; phone 631-344-3004; fax 631:344-3374 
CZT detectors entails the ability to grow large uniform single crystals, to improve procedures for fabricating the devices, and to ensure a better, more uniform response.

Characterization of the detectors, along with detailed investigations of their spatial response will help to resolve these issues. Such studies; undertaken by several groups, have delineated the detrimental effects of grain boundaries and twins, but could not describe the physical mechanisms responsible for carrier trapping [1-4]. Since these techniques relied upon standard laboratory gammaray: sources"or alpha particles, they had limited capability for characterizing the response at high spatial-resolution. Compared to these techniques, synchrotron radiation offers the advantages of high brightness and collimation with the capability of a tunable energy to study either bulk or surface effects. At BNL's National Synchrotron Light Source (NSLS), we developed a unique experimental. setup "with a spatial resolution better than $10 \mu \mathrm{m}$. With this setup, we carried out a series of investigations on CZT detectors, giving us new insights into the critical role of small-scale defects (i.e., tellurium-rich inclusions) on the detector's efficiency and energy resolution : 5]. As shown in fig. 1, the probe used to spatially investigate the detector's response was critical in revealing the nonuniformity of collected charge that corresponded to the abundance of the Te-rich secondary phases, and thus clearly demonstrated their deleterious effect.

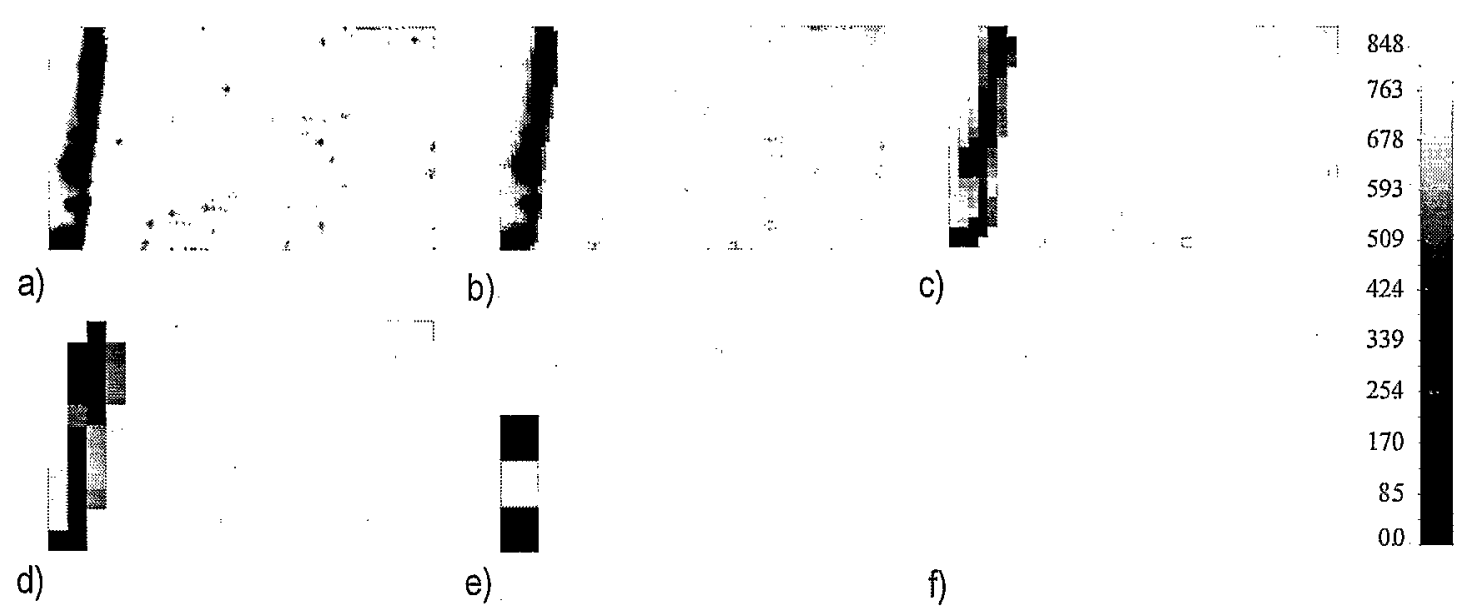

Fig. 1. Six maps showing the $x$-ray response of the same device area evaluated with different spatial resolutions to simulate" different beam sizes: a) $10 \times 10$, b) $20 \times 20$, c) $50 \times 50$, d) $100 \times 100$; e) $200 \times 200$, and, f) $400 \times 400 \cdot \mu \mathrm{m}^{2}$. The original $\mathrm{x}$-ray map was measured with $10-\mu \mathrm{m}$ steps in both directions with the beam size of $10 \times 10 \mu \mathrm{m}^{2}$. To generate the other: maps, the pulse-height spectra measured for several adjacent beam locations $(2 \times 2,4 \times 4$; etc.) were added together, and new peak positions were determined by using a Gaussian fit.

Recently, a dedicated beam-line was developed with improved resolution that will assist us in gaining an understanding of, and modeling the mechanism involving Te-rich secondary phases and charge loss. In . addition, an IR system with counting capability allows us to correlate the concentration of defects and the performances of large volume CZT devices. [6]. In this paper, after briefly describing the systems, several experimental results are presented showing the effect of Te inclusions (size, concentration, and spatial distribution) on the effectiveness of CZT detectors. 


\section{EXPERIMENTAL SETUPS}

Based on the experience gained during our previous experiments [5, 7], a new end-station was established and a dedicated beam-line is now operating and available for studies on semiconductor radiation detectors. The energy of the $\mathrm{x}$-ray source ranges between 1 and $100 \mathrm{keV}$, and a doublechannel-cut monochromator enables the selection of photons between 8 and $40 \mathrm{keV}$. As the schematic in fig. 2a shows, the original size of the synchrotron beam is reduced to $1 \times 1 \mathrm{~mm}^{2}$ through a cooled aperture, and its intensity is monitored with an ion chamber. The mapping system consists of a positioning platform, data-acquisition hardware, and control system. The platform is a set: of $x-y-z$ translation stages with sub-micron : resolution that permits raster scanning with high precision and repeatability. The system is equipped with two tungsten double-slit collimators that, respectively, reduce the beam's size to below: $10 \times 10 \mu \mathrm{m}^{2}$ and $3 \times 3 \mu \mathrm{m}^{2}$ (fig. $2 \mathrm{~b}$ ). Its position with respect to the incident beam is controlled to sub-micron spatial resolution by two motorized $y-z$ translation stages.

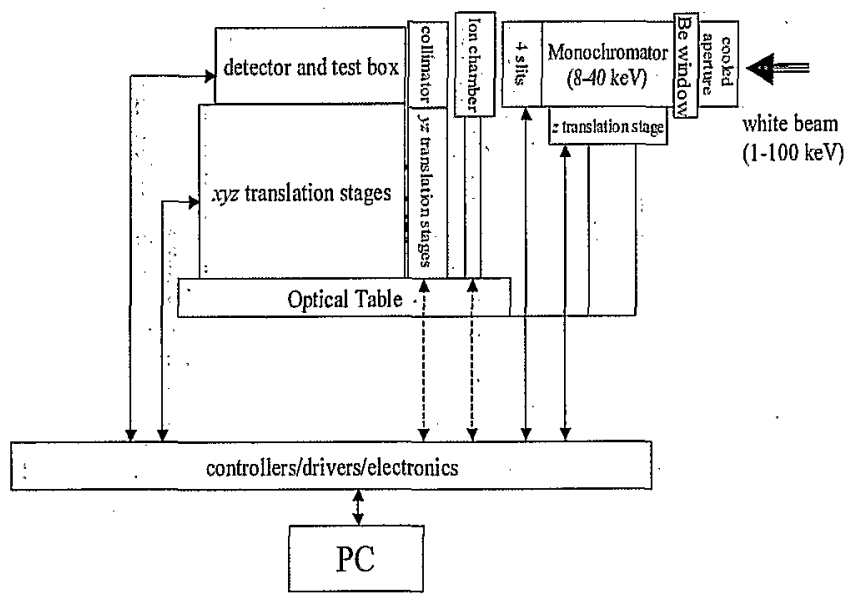

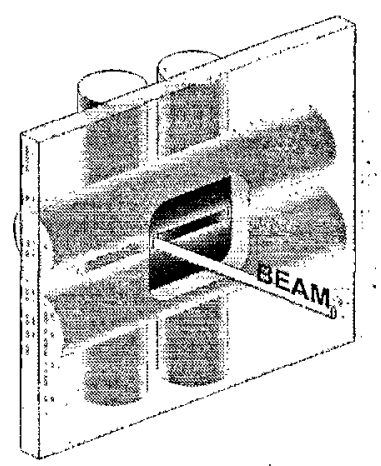

b)

Fig. 2: a). Block diagram showing the mapping system. b) Sketch of the collimators: both are composed of a pair of crossed slits. The slits are realized by spacing, with a calibrated film; two tungsten rods of the appropriate size.

There are several options for mounting CZT samples or other devices. For example, small samples can be mounted in a standard eV-Products' holder with a Be window attached to a preamplifier box [8]. The output signals from the charge-sensitive preamplifiers (waveforms) also can be recorded with a multi-channel oscilloscope, offering another possible way to look at the detector's response: In principle, any small semiconductor detector (within a few $\mathrm{cm}^{2}$ ) with a proper readout can be studied at the energy of interest.

A SPEC user interface (a UNIX-based software package developed for x-ray diffraction) controls the motors and the data-acquisition system [9]. At each point of the raster scan, a pulseheight spectrum; corresponding to the area of the detector irradiated at the cathode side, is recorded through a multi-channel analyzer (MCA), and stored for further processing. For the energies typically 
used for our measurements, $20-30 \mathrm{keV}$, the flux is in the range $10^{3}-10^{4}$ photons $/ \mathrm{sec}$ and good statistics can be achieved within 1-3 s acquisition time per point, depending on the resolution adopted; so that large areas can be scanned within an acceptable time. For instance the scan of $1 \times 1$ $\mathrm{mm}^{2}$ with $10-\mu \mathrm{m}$ resolution requires less than 3 hours:

This x-ray system is intended for studying the physics of CZT. detectors, in particular the mechanism of charge transport; to give crystal growers new insights; and to refine the development of a theoretical model [10] that could lead to improvements in the device's design and fabrication: processes.

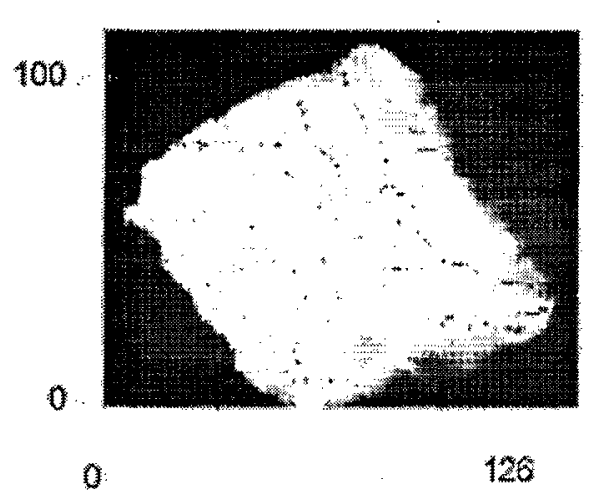

a)

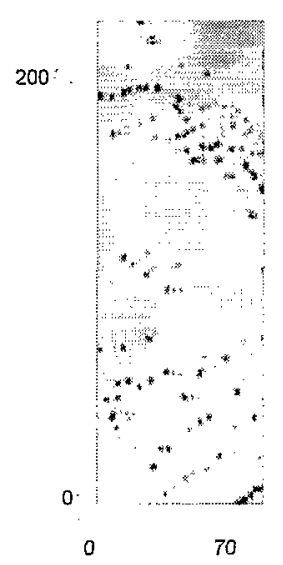

b)

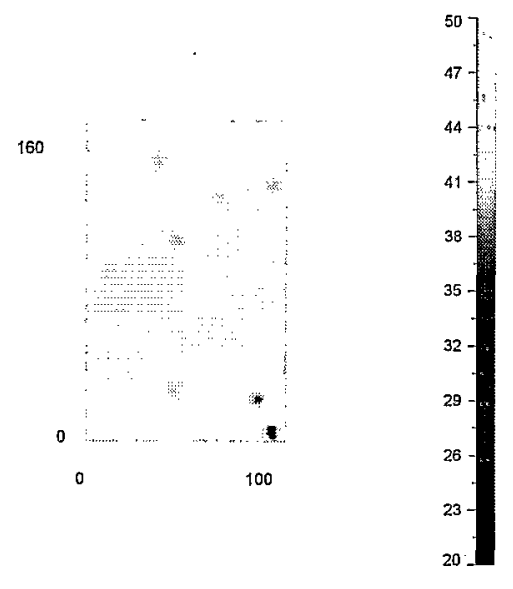

c)

Fig. 3. 2-D maps of peak position for a 1-mm thick detectors. The scans were carried out using:20-keV x-rays with different resolutions. To optimize the characterization; the material was first scanned with a $10 \mathrm{x} 10 \mu \mathrm{m}^{2}$. beam and $50 \mu \mathrm{m}$ step-size to investigate the response of the full detector (a). High dislocation fields decorated with Te inclusions can be observed, along with other non-uniformities related to surface treatment. Afterwards, smaller regions showing interesting features: are selected for further scans with improved resolution, as shown in (b) $10 \times 10 \mu \mathrm{m}^{2}$ beam and $10 \mu \mathrm{m}$ step-sizes, and (c) $3 \times 3 \mu \mathrm{m}^{2}$. beam and $3 \mu \mathrm{m}$ step-sizes.

To quickly screen CZT crystals and obtain quantitative information relating the size and. concentrations of Te inclusions to the device's performance, we employed an automatic IR transmission microscope system. The system; that will be described elsewhere [6], includes an illuminating source, a positioning platform consisting of a set of $x-y-z$ translation stages, and a large field-of-view microscope coupled with a $3.5-\mu \mathrm{m}$ pixel-size digital camera. The $\sim 3000 \times 2000$-pixel images have a: resolution of $0: 19$ - and $0.75-\mu \mathrm{m}$ per pixel, depending on the optical magnification of the lens used. To determine size and shape of the detected Te-rich secondary phases, an iterative algorithm: was developed. The samples are probed in a fixed number of points; hence, for each chosen position, a set of images is taken at different depths to picture layers $\sim 50-100 \mu \mathrm{m}$ distant from each other: During data analysis, Te inclusions identified within each layer are subtracted from the total counts seen in several images. The final results are depicted in histograms of concentrations versus sizes. 


\section{RESULTS AND DISCUSSION}

The typical experiment exploring the uniformity of charge-collection efficiency of CZT detectors consists of obtaining raster scans on 1-mm thick planar devices to probe their spatial response to hard $x$-rays (fig. 3). In the range of energies of interest, the linear absorption length: for detector-grade CZT material $(10 \% \mathrm{Zn})$ is about a few hundred microns. Electron-hole pairs created by the absorption of x-rays drift from the cathode to the anode under the influence of the applied electric field. These charges experience diffusion and mutual electrostatic repulsion [11], both of which broaden the electron cloud. However, for the typical energies and electric fields used during. these studies, the effects of former are more significant:than the latter [10]: The amount of collected. charge corresponds to the response of the small volume under investigation. Therefore, information on the device's response to the ionization events locally produced by the highly collimated beam is mirrored in the shapes of the spectra and the waveforms. Usually, the peak positions in the spectra versus the beam locations are used to generate a 2-dimensional map of the device's response, which reflects the local material properties of the detector.

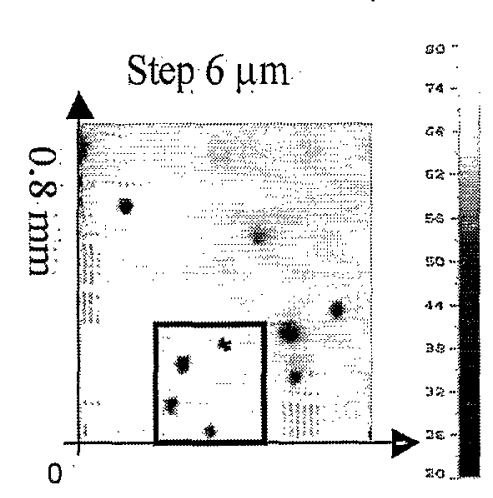

a)

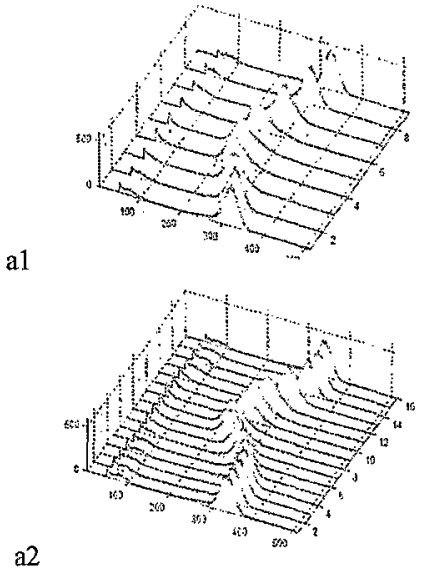

b)

)

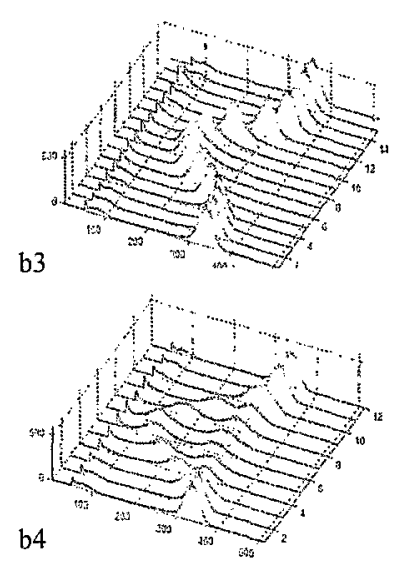

b4

Fig. 4. a) 2-D map of the peak position: scan performed with a 6- $\mu \mathrm{m}$ step in both directions. b) Spectra along lines crossing through the center of the selected spots. The lines were horizontal to the map. Each spectrum corresponds to a 6$\mu \mathrm{m}$ distance.

When electrons encounter a crystalline defect during their drift toward the anode, an unpredictable amount of charges can become trapped. Te inclusions (possibly including the areas of relatively high dislocations surrounding the defects) trap electrons, but in a different manner to the point traps (e.g., Cd vacancies) normally present in CZT. The difference is related to their distribution and morphology. Thus, in contrast to randomly distributed point traps, these Te inclusions are extended defects (over distances of tens of microns or more) with a very high concentration of local trapping centers. Moreover, we note that any electric-field distortions around these defects also can: 
contribute to dispersing the collected charges and to degrading the uniformity of the device's response and spectroscopic performance.

Te inclusions are expected to affect charge collection differently depending on their size and position viz. the electrodes. In fact, while drifting towards the anode, electron clouds experience diffusion and electrostatic repulsion, i.e., they grow and broaden depending on the deposited ionizing energy and applied electric field. If we consider a Te inclusion as a spherical volume trapping all charges impinging on it [10], the efficiency of charge collection is less affected by defects that are located an appreciable distance away from the interaction region because a smaller fraction of the charges will collide with them. Thus, for instance, Te inclusions closer to the anode are responsible. for less charge loss than those further away. The results shown in fig. 4 confirm this assumption.
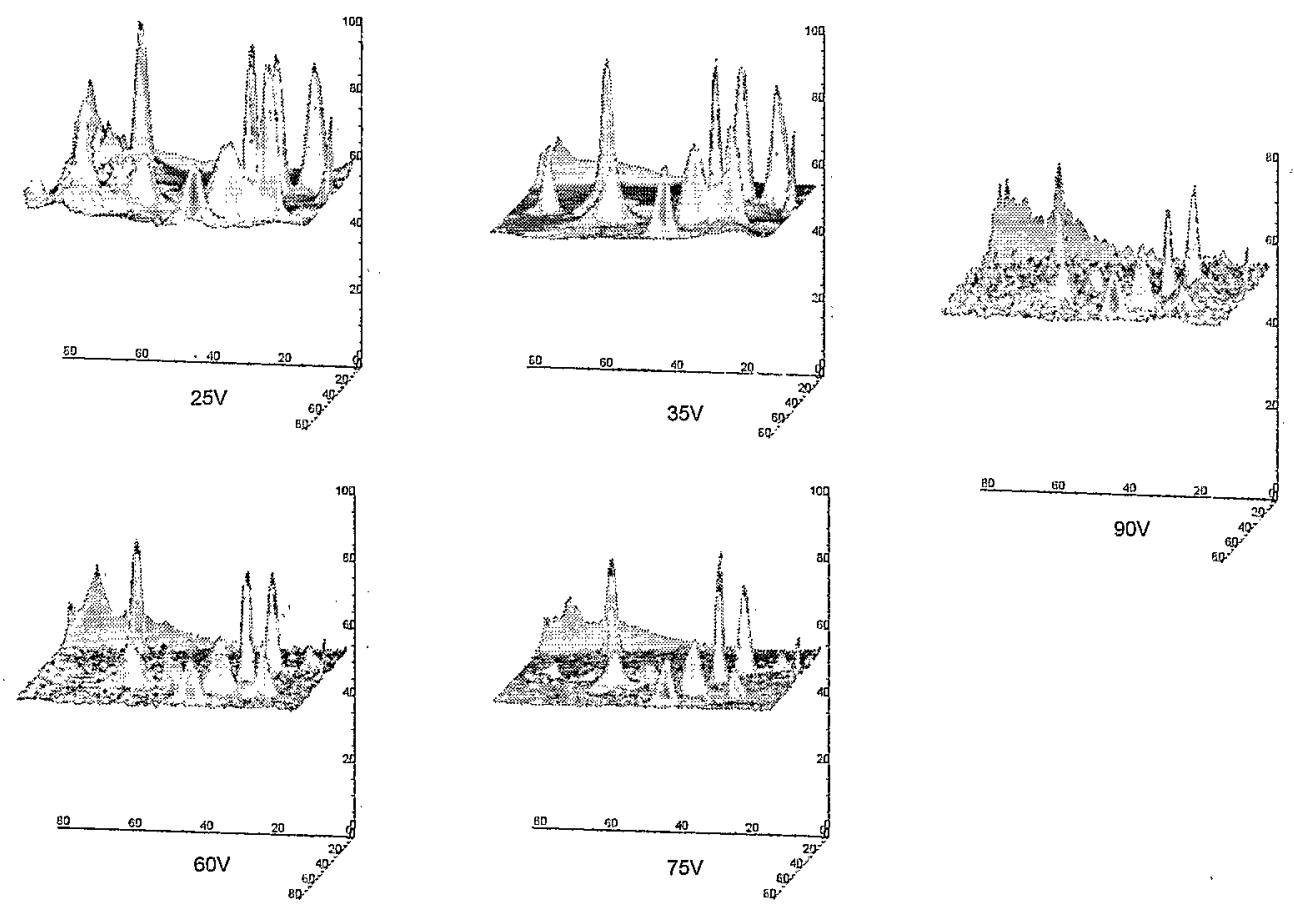

Fig. 5: 10- $\mu \mathrm{m}$ resolution maps of the charge-collection efficiency in the same region at different bias voltages.

Spectra analysis was performed in the region enclosed by the box: the two "dark" spots in the upper side of this box (b3 and b4 from the left) were located in an IR image at the cathode side; the two in the lower side (a1: and a2 from the right) were found at a depth of $\sim 500 \mu \mathrm{m}$ below the cathode, corresponding to the middle of the crystal: A clear correlation between the depth of the inclusion and its effect on the $\mathrm{X}$-ray response is apparent from the IR images and spectra analysis. In particular, spectra corresponding to defects located near the cathode in an IR image (b3, b4) show a much lower peak position; indicative of more severe charge losses, as well as double peaks in the energy spectra, than do defects located deeper in the crystal $(\sim 500 \mu \mathrm{m})$ that cause the photo-peak to broaden and shift to lower position (a1, a2). Further investigations are needed with very detailed IR characterization 
and precise correlation with the x-ray scans to conclusively determine the precise extent to which a defect's position affects charge collection.

Another important factor to be investigated is the influence of the applied electric field. To reduce the effect of standard defects; the drift time $\left(t_{d}\right)$ must be much smaller than the life time $(\tau)$. Thus, to further investigate the mechanism leading to the local deterioration of the $\mathrm{x}$-ray response, several scans were taken of the same area of the detector at different bias voltages; Figure 5 contains the resulting maps: To better highlight their differences, the maps were plotted upside down, to fully reveal the variation of the non-uniformities corresponding to regions surrounding the Te inclusions with the applied electric field.

The degraded areas become smaller as the voltage increases, until some seem to disappear at higher voltages. Assuming that the electron-cloud broadening lessens with an increasing electric field [10], the spatial effect of the inclusions is diminished for smaller electron clouds. However, it is unclear by how. much the peak position is shifted with the applied electric field, and further investigations are required that take into account the eventual non-uniformity of the electric field due to the presence of inclusions.

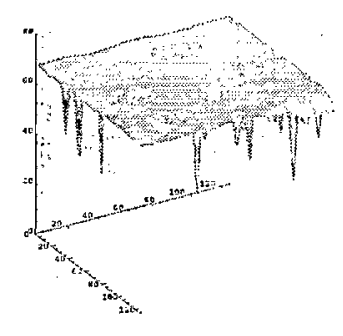

a)

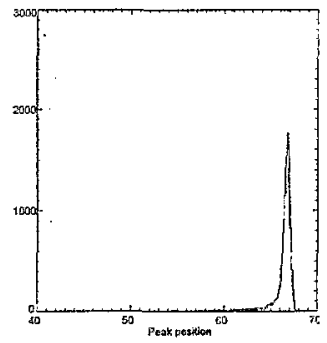

)

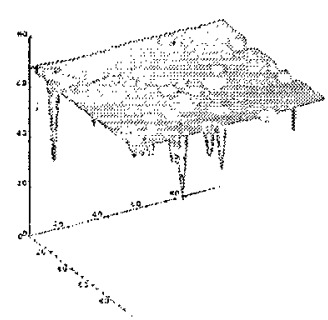

b)

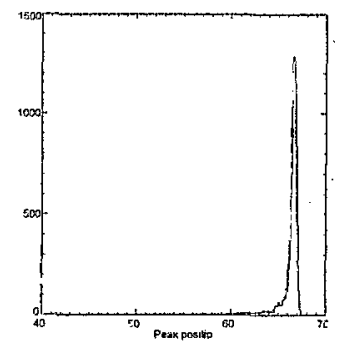

Fig. 6.3-D maps of peak positions and histograms of the peak positions corresponding to the total area of the map. a) 1.27 x 1:27. $\mathrm{mm}^{2}$ pixel; b) $1 \times 1 \mathrm{~mm}^{2}$ pixel.

As discussed, having the ability to obtain such a detailed characterization of detector's response is .very promising for our understanding. of the mechanisms of charge trapping in semiconductor devices. So far; this technique has allowed us to draw an important conclusion inherent the observed spatial variations in the charge-collection efficiency, which severely limits the performance of long-drift-length, large-volume CZT detectors [5]. This is, the fluctuations of charge loss caused by trapping centers associated with the Te inclusions, i.e., the cumulative effect of the $\mathrm{Te}$ inclusions, account for the limited spectroscopic performances.

To demonstrate the fluctuations of the collected charge corresponding to small volumes rich in Te inclusions, maps of the peak position for several small areas were considered to simulate the situation corresponding to $1-\mathrm{mm}$ layer of a pixilated device. First, a scan was carried out with a 30$\mathrm{keV}$ beam and a $10-\mu \mathrm{m}$ resolution on a detector biased under $\sim 500 \mathrm{~V} / \mathrm{cm}$ electric field. Then, pixel sizes ranging between $0.5 \times 0.5$ and $1.5 \times 1.5 \mathrm{~mm}^{2}$ were simulated. The results can be seen in figs. 6 and 7. Beside the 3-D representations of the peak position versus the spatial position, histograms of their values are shown. They illustrate the fluctuations of the photopeak position in areas containing several defects. The crystal used for this detector exhibited a random distribution of Te inclusions 
between 15 - and $25-\mu \mathrm{m}$ diameter. It seems clear, especially from the findings in fig. 7 from several adjacent voxels of $0.5 \times 0.5 \times 1 \mathrm{~mm}^{3}$, that the performance of a single voxel strongly depends on the concentration, size, and position of Te inclusions within it, leading to unpredictable fluctuations and requiring a very refined correction scheme: According to these results, the performances of thick devices are:limited by the observed fluctuation of the collected charge that becomes more extensive when electron clouds drift longer toward the anodes typically encountering similar distributions of defects.

In the case of Frisch-ring detectors $[12,13]$, we observed poor energy resolution for crystals showing a high concentration of Te inclusions of $\sim 10-30 \mu \mathrm{m}$ size. For instance, a detector with a $9.8 \mathrm{E}+04$ inclusions $/ \mathrm{cm}^{3}$ exhibited $6.2 \%$ FWHM at $662 \mathrm{keV}$. Following this new information, crystal growers are working to produce crystals with fewer Te inclusions. Crystals with $2-5 \mathrm{um} \mathrm{Te}$ inclusions at a concentration of $1.2 \mathrm{E}+06$ inclusions $/ \mathrm{cm}^{3}$ already have been tested in the Frisch-ring configuration; and exhibited a resolution better than $0.8 \%$ at $662 \mathrm{keV}$, even for drift lengths of $10-11$ $\mathrm{mm}$. Considering the extremely simple configuration of such kind of detectors, this result bodes well for the future development of CZT devices.

\section{CONCLUSION}

Spatial micro-characterization of CZT detectors employing synchrotron radiation as a probe helped to understand some material properties limiting the performances of CZT detectors. The role of Te inclusions. was investigated and their effect in limiting detector's spectroscopic performances demonstrated. The size and concentration of the defects, along.with the device's thickness and photon energy are the key factors in predicting the detectors' performances. A dedicated beam-line for investigating the spatial response of semiconductor detectors was developed and is operating at BNL's National Synchrotron Light Source. This tool will help in establishing better material growth and fabrication techniques; and possibly, for designing detectors. Moreover, to correlate information about the concentration andisize of the Te inclusions: with the performances of thick; large-volume devices, we developed an IR system with counting capability. A close feedback with industrial suppliers was set up to inform them of our findings; so they could correct growth- and fabricationrelated problems and produce better detector modules.

\section{ACKNOWLEDGEMENTS}

This:work was.supported by U.S. Department of Energy, Office of Nonproliferation Research and Engineering, NA-22. The manuscript has been authored by Brookhaven Science Associates, LLC under Contract ${ }^{-}$No. DE-AC02-98CH10886 with the U.S. Department of Energy. The United States Government retains, and the publisher, by accepting the article for publication, acknowledges, a world-wide license to publish or reproduce the published form of this manuscript, or allow others to do so, for the United States Government purposes.

The authors wish to acknowledge the support of the technical and scientific NSLS staff, in particular D. P. Siddons, W. Caliebe, Z. Zhong, Z. Yin, T. Lenhard, S. Cheung, and D. Poshka. We are also grateful to F. Cetero, I. Dioszegi, W. Licciardi and G. Mahler. 


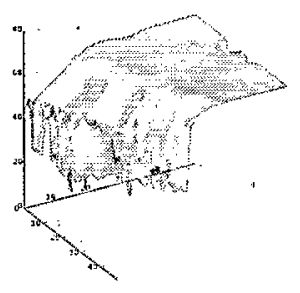

pixel 1

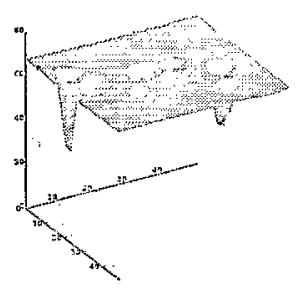

pixel 3 .
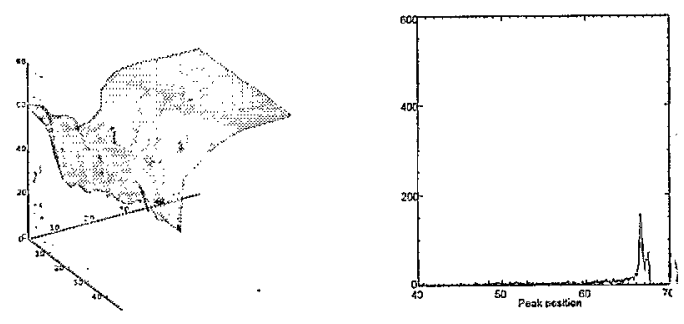

pixel_5
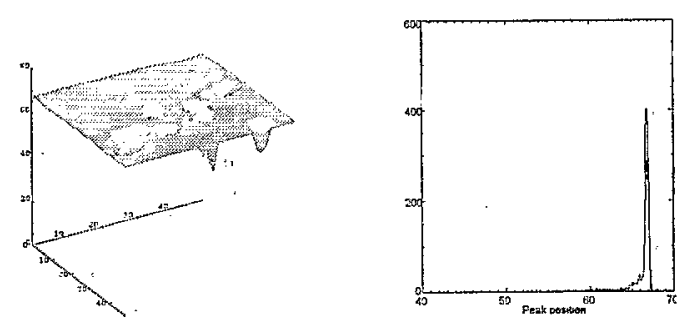

pixel_7

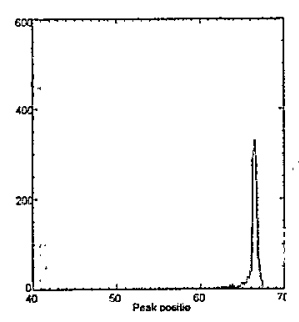

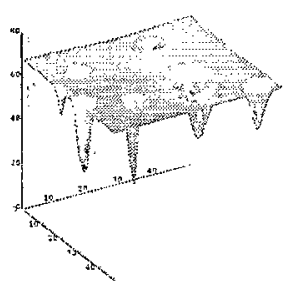

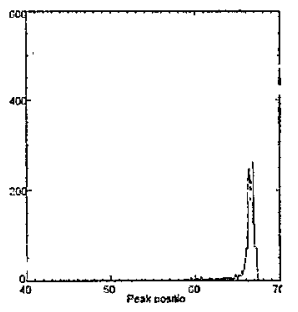

pixel_2
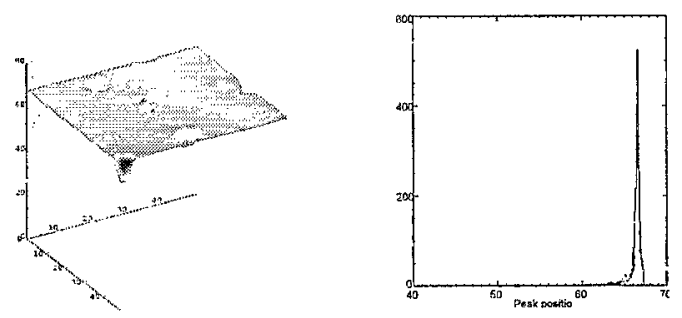

pixel_4
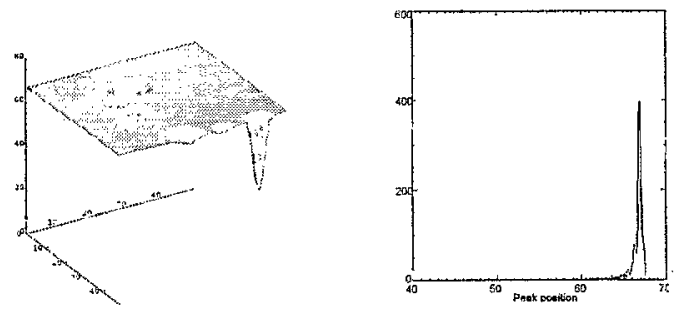

pixel_6
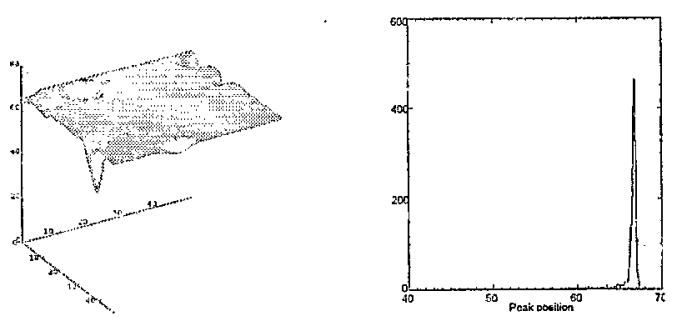

pixel_8

Fig. 7. 3-D maps of peak positions and histograms of the peak positions corresponding to the total area of the map for eight adjacent $0.5 \times 0.5 \mathrm{~mm}^{2}$ pixels. 


\section{REFERENCES}

[1] B. A. Brunett; J. M. Van Scyoc, N. R. Hilton, J. C.. Lund, R. B. James, and T. E. Schlesinger, IEEE Trans.. Nucl. Sci. 46; 237,1999 .

[2] B: A.: Brunett; J. M. Van Scyoc, T. E. Schlesinger, and R. B. James, Nucl. Instr. Meth. A458, 76, 2001.

[3] M. Amman, J. S. Lee, and P. N. Luke, Proc. SPIE 4507, 1, 2001.

[4] P: N. Luke; M. Amman, and J. S. Lee, IEEE Trans. Nucl. Sci. 51, 1199, 2004.

[5] G. A: Carini, A. E. Bolotnikov, G. S. Camarda; G. W. Wright, L. Li, and R. B. James, Appl. Phys. Lett. 88, 143515, 2006:

[6] G. S. Camarda, A. E. Bolotnikov, G. A. Carini, Y. Cui, K. T. Kohman, L. Li, and R. B. James, SPIE 6319A, San Diego 2006 .

[7] G. A. Carini, A. E. Bolotnikov, G. S. Camarda, G. W. Wright, G. De Geronimo, D. P. Siddons, and R. B. James, IEEE Träns: Nucl. Sci. 52, 1941, 2005.

[8]: eV·Products, a division of II-VI Inc., Saxombourg; PA 16056.

[9] http://www.certif:com.

[10]A. E.:Bolotnikov, G. S. Camarda, G. A. Carini, Y. Cui, L. Li, and R. B. James, submitted to Nucl. Instr. Meth. A.

[11]E: Gatti, A. Longoni, P. Rehak, and M. Sampietro; Nucl. Instr. Meth. A253, 393, 1987.

[12].W. J. McNeil, D: S. McGregor, A. E: Bolotnikov, G: W. Wright, and R. B: James, Appl. Phys. Lett. 84, 1988, 2004.

[13]A. E. Bolotnikov, G. C..Camarda, G. A. Carini, M. Fiederle; L. Li, D. S. McGregor; W. McNeil, G. W: Wright; and R. B. James, IEEE Trans: Nucl. Sci. 53, 2006. 